\title{
The Hierarchy of Hamiltonians for a Restricted Class of Natanzon Potentials
}

\author{
Elso Drigo Filho ${ }^{a *}$ and Regina Maria Ricotta ${ }^{b}$ \\ ${ }^{a}$ Instituto de Biociências, Letras e Ciências Exatas, IBILCE-UNESP \\ Rua Cristovão Colombo, 2265, 15054-000 São José do Rio Preto, SP, Brazil \\ ${ }^{b}$ Faculdade de Tecnologia de São Paulo, FATEC/SP-CEETPS-UNESP \\ Praça Fernando Prestes, 30, 01124-060, São Paulo, SP, Brazil
}

Received on 10 November, 2000. Revised version received on 10 January, 2001

\begin{abstract}
The restricted class of Natanzon potentials with two free parameters is studied within the context of Supersymmetric Quantum Mechanics. The hierarchy of Hamiltonians and a general form for the superpotential is presented. The first members of the superfamily are explicitly evaluated.
\end{abstract}

\section{Introduction}

The classes of Natanzon potentials, namely, the hypergeometric and the confluent, reffer to potentials whose Schrödinger equation is analytically and exactly solvable by means of hypergeometric functions. They have motivated several works concerning the mathematical and algebraic aspects of their structure and solutions and have numerous applications in several branches of physics, [1]-[7].

In particular, there have been studies within Supersymmetric Quantum Mechanics formalism. Cooper et al, [6], for instance, investigated the relationship between shape invariance and exactly analytical solvable potentials and showed that the Natanzon potential is not shape invariant although it has analytical solutions for the associated Schrödinger equation. Lévai et al, [7], have determined phase-equivalent potentials for a class of Natanzon potentials employing the formalism of supersymmetry.

However, the hierarchy of the Hamiltonians corresponding to Natanzon potentials has not been determined yet. In ref. [6], Cooper et al have sketched the first few potentials of the hierarchy from the knowledge of their asymptotic behaviour from the series approximation. In this paper we construct the hierarchy of Hamiltonians of the restricted class of Natanzon potentials, (Ginocchio class), with two free parameters. The first few members of the superfamily are explicitly evaluated and a general form for the superportential is proposed by induction.

\section{Supersymmetric Quantum Mechanics Formalism}

In the formalism of Supersymmetric Quantum Mechanics there are two operators $Q$ and $Q^{+}$, that satisfy the algebra

$$
\{Q, Q\}=\left\{Q^{+}, Q^{+}\right\}=0, \quad\left\{Q, Q^{+}\right\}=H_{S S}
$$

where $H_{S S}$ is the supersymmetric Hamiltonian. The usual realisation of the operators $Q$ and $Q^{+}$is

$$
\begin{gathered}
Q=a_{1}^{-} \sigma^{-}=\left(\begin{array}{cc}
0 & 0 \\
A^{-} & 0
\end{array}\right) \\
Q^{+}=a_{1}^{+} \sigma^{+}=\left(\begin{array}{cc}
0 & A^{+} \\
0 & 0
\end{array}\right)
\end{gathered}
$$

where $\sigma^{ \pm}$are written in terms of the Pauli matrices and $A^{ \pm}$are bosonic operators. With this realisation the supersymmetric Hamiltonian $H_{S S}$ is given by

$$
H_{S S}=\left(\begin{array}{cc}
A^{+} A^{-} & 0 \\
0 & A^{-} A^{+}
\end{array}\right)=\left(\begin{array}{cc}
H^{+} & 0 \\
0 & H^{-}
\end{array}\right) \text {. }
$$

where $H^{ \pm}$are supersymmetric partner Hamiltonians and share the same spectra, apart from the nondegenerate ground state. Using the super-algebra a given

\footnotetext{
${ }^{*}$ Work supported in part by $\mathrm{CNPq}$
} 
Hamiltonian can be factorized in terms of the bosonic operators. In $\hbar=c=1$ units, it is given by

$$
H_{1}=-\frac{d^{2}}{d r^{2}}+V_{1}(r)=A_{1}^{+} A_{1}^{-}+E_{0}^{(1)}
$$

where $E_{0}^{(1)}$ is the lowest eigenvalue. The bosonic operators are defined by

$$
A_{1}^{ \pm}=\left(\mp \frac{d}{d r}+W_{1}(r)\right)
$$

where the superpotential $W_{1}(r)$ satisfies the Riccati equation

$$
W_{1}^{2}-W_{1}^{\prime}=V_{1}(r)-E_{0}^{(1)}
$$

The eigenfunction for the lowest state is related to the superpotential $W$ as

$$
\Psi_{0}^{(1)}(r)=N \exp \left(-\int_{0}^{r} W_{1}(\bar{r}) d \bar{r}\right)
$$

or conversely

$$
W_{1}(r)=-\frac{d}{d r} \ln \left(\Psi_{0}^{(1)}\right)
$$

Now it is possible to construct the supersymmetric partner Hamiltonian,

$$
H_{2}=A_{1}^{-} A_{1}^{+}+E_{0}^{(1)}=-\frac{d^{2}}{d r^{2}}+\left(W_{1}^{2}+W_{1}^{\prime}\right)+E_{0}^{(1)} .
$$

If one factorizes $H_{2}$ in terms of a new pair of bosonic operators, $A_{2}^{ \pm}$one gets,

$$
H_{2}=A_{2}^{+} A_{2}^{-}+E_{0}^{(2)}=-\frac{d^{2}}{d r^{2}}+\left(W_{2}^{2}-W_{2}^{\prime}\right)+E_{0}^{(2)}
$$

where $E_{0}^{(2)}$ is the lowest eigenvalue of $H_{2}$ and $W_{2}$ satisfy the Riccati equation,

$$
W_{2}^{2}-W_{2}^{\prime}=V_{2}(r)-E_{0}^{(2)} .
$$

Thus a whole hierarchy of Hamiltonians can be constructed, with simple relations connecting the eigenvalues and eigenfunctions of the $n$-members, [8]-[13]

$$
\begin{gathered}
H_{n}=A_{n}^{+} A_{n}^{-}+E_{0}^{(n)} \\
A_{n}^{ \pm}=\left(\mp \frac{d}{d r}+W_{n}(r)\right)
\end{gathered}
$$

$$
\begin{gathered}
\Psi_{n}^{(1)}=A_{1}^{+} A_{2}^{+} \ldots \psi_{0}^{(n+1)} \quad E_{n}^{(1)}=E_{0}^{(n+1)} \\
\Psi_{0}^{(1)}(r)=N \exp \left(-\int_{0}^{r} W_{1}(\bar{r}) d \bar{r}\right) .
\end{gathered}
$$

\section{Natanzon Potential and the Hierarchy of Hamiltonians}

The restricted class of Natanzon potentials having two parameters and given in terms of the variable $y(r)$ is,

$$
V(r)=\left\{-\lambda^{2} v(v+1)+1 / 4\left(1-\lambda^{2}\right)\left[5\left(1-\lambda^{2}\right) y^{4}-\left(7-\lambda^{2}\right) y^{2}+2\right]\right\}\left(1-y^{2}\right),
$$

where the variable function $y(r)$ satisfies $d y / d r=\left(1-y^{2}\right)\left[1-\left(1-\lambda^{2}\right) y^{2}\right]$. The dimensionless free parameters $v$ and $\lambda$ measure the depth and the shape of the potential, respectively.

The Schrödinger equation for this potential, [2], [3], in dimensionless units, is given by

$$
\left[-d^{2} / d r^{2}+V(r)\right] \Psi_{n}(r)=\epsilon_{n} \Psi_{n}(r)
$$

where $V(x)=v_{0} V(r), \epsilon_{n}=E_{n} / v_{0}$ and $r=b x=\left(2 m v_{0} / \hbar^{2}\right)^{1 / 2} x$.

The analytic solutions for the energy eigenfunctions are given by,

$$
\Psi_{n}=\left(1-\lambda^{2}\right)^{\mu_{n} / 2}[g(y)]^{-\left(2 \mu_{n}+1\right) / 4} C_{n}^{\mu_{n}+1 / 2}\left(\lambda y /[g(y)]^{1 / 2}\right)
$$


where $g(y)=1-\left(1-\lambda^{2}\right) y^{2}$. The factor $C_{n}^{(a)}(x)$ is a Gegenbauer polynomial when $n$ is a non-negative integer, which is our case. The corresponding energy eigenvalues are given by $\epsilon_{n}=-\mu_{n}^{2} \lambda^{4}, \quad \mu_{n}>0$, where

$$
\mu_{n} \lambda^{2}=\left[\lambda^{2}(v+1 / 2)^{2}+\left(1-\lambda^{2}\right)(n+1 / 2)^{2}\right]^{1 / 2}-(n+1 / 2)
$$

Notice the relationship between the energy levels which will be extensively used in what follows,

$$
\left(\mu_{n}^{2}-\mu_{n-1}^{2}\right) \lambda^{2}=\left(-2 n-(2 n+1) \mu_{n}+(2 n-1) \mu_{n-1}\right) .
$$

In order to construct the superfamily we firstly factorize the Natanzon potential, calling $V(r)=V_{1}(r)=$ $V_{-}(r)+\epsilon_{0}^{(1)},[6]$. The factorized Schrödinger equation is given by

$$
H_{1}-\epsilon_{0}^{(1)}=a_{1}^{+} a_{1}^{-}, \quad a_{1}^{ \pm}=\mp d / d r+W_{1}(r)
$$

where $\epsilon_{n}^{(1)}=\epsilon_{n}$. The superpotential $W_{1}(r)$ is evaluated from the knowledge of the ground state eigenfunction of $V(r)$ by using (8) with $\Psi_{n}^{(1)}=\Psi_{n}$, given by (18). It satisfies the Riccati equation and it is given by

$$
W_{1}(r)=\left(1-\lambda^{2}\right) y\left(y^{2}-1\right) / 2+y \mu_{0} \lambda^{2} .
$$

The superpartner Hamiltonian satisfies the equation

$$
H_{2}-\epsilon_{0}^{(1)}=a_{1}^{-} a_{1}^{+}
$$

which is written in terms of $V_{2}(r)$ as

$$
W_{1}^{2}+W_{1}^{\prime}=V_{2}(r)-\epsilon_{0}^{(1)}
$$

where $V_{2}(r)$, the potential for the second member of the hierarchy, is given by

$$
V_{2}(r)=\left\{-\mu_{0}^{2} \lambda^{4}+\mu_{0} \lambda^{2}+1 / 4\left(1-\lambda^{2}\right)\left[-7\left(1-\lambda^{2}\right) y^{4}+\left(9-3 \lambda^{2}-8 \mu_{0} \lambda^{2}\right) y^{2}-2\right]\right\}\left(1-y^{2}\right) .
$$

To construct the next member of the superfamily, we factorize the Schrödinger equation for $V_{2}$. It gives

$$
H_{2}-\epsilon_{0}^{(2)}=a_{2}^{+} a_{2}^{-}, \quad a_{2}^{ \pm}=\mp d / d r+W_{2}(r)
$$

where $W_{2}(r)$ satisfies the associated Riccati equation,

$$
W_{2}^{2}-W_{2}^{\prime}=V_{2}(r)-\epsilon_{0}^{(2)} .
$$

$\epsilon_{0}^{(2)}$ is the energy ground state of the potential $V_{2}(r)$ and it is such that $\epsilon_{0}^{(2)}=\epsilon_{1}^{(1)}$. The superpotential $W_{2}$ can be computed from the ground state wave function $\Psi_{0}^{(2)}$. It is given by $W_{2}(r)=-\frac{d}{d r} \log \left(\Psi_{0}^{(2)}\right)$, where $\Psi_{0}^{(2)}=a_{1}^{-} \psi_{1}^{(1)}$, i.e.,

$$
W_{2}(r)=3 / 2\left(1-\lambda^{2}\right) y\left(y^{2}-1\right)+y \mu_{1} \lambda^{2}-\frac{d}{d r} \log \left(f_{1}\right)
$$

where

$$
f_{1}(y)=1+a_{11} y^{2}
$$

and the coefficient $a_{11}$ is given by

$$
a_{11}=\left(\mu_{0}-\mu_{1}\right) \lambda^{2}-1 \text {. }
$$

The new superpartner of $\mathrm{H}_{2}$ is given by

$$
W_{2}^{2}+W_{2}^{\prime}=V_{3}(r)-\epsilon_{0}^{(2)}
$$

where $V_{3}(r)$, the potential for the third member of the hierarchy, is given by

$$
\begin{aligned}
V_{3}(r) & =\left\{-\mu_{1}^{2} \lambda^{4}+\mu_{1} \lambda^{2}+1 / 4\left(1-\lambda^{2}\right)\left(-27\left(1-\lambda^{2}\right) y^{4}+\left(33-15 \lambda^{2}\right) y^{2}-6\right)+\right. \\
& \left.+\frac{2 a_{11} g(y)}{f_{1}(y)}\left\{1+\left(-9+6 \lambda^{2}-2 \mu_{1} \lambda^{2}\right) y^{2}+8\left(1-\lambda^{2}\right) y^{4}\right\}+8 a_{11}^{2} y^{2}\left(1-y^{2}\right)\left(\frac{g(y)}{f_{1}(y)}\right)^{2}\right\}\left(1-y^{2}\right)
\end{aligned}
$$


and $g(y)=1-\left(1-\lambda^{2}\right) y^{2}$. Thus, factorizing the Hamiltonian for this potential we have

$$
H_{3}-\epsilon_{0}^{(3)}=a_{3}^{+} a_{3}^{-}, \quad a_{3}^{ \pm}=\mp d / d r+W_{3}(r)
$$

where $W_{3}(r)$ satisfies the Riccati equation,

$$
W_{3}^{2}-W_{3}^{\prime}=V_{3}(r)-\epsilon_{0}^{(3)} .
$$

$\epsilon_{0}^{(3)}$ is the energy ground state of the potential $V_{3}(r)$, with $\epsilon_{0}^{(3)}=\epsilon_{1}^{(2)}=\epsilon_{2}^{(1)}$. Again, the superpotential $W_{3}$ can be computed from the ground state wave function $\Psi_{0}^{(3)}$, defined by $W_{3}(r)=-\frac{d}{d r} \log \left(\Psi_{0}^{(3)}\right)$, with $\Psi_{0}^{(3)}=a_{2}^{-} a_{1}^{-} \psi_{2}^{(1)}$. It is given by

$$
W_{3}=5 / 2\left(1-\lambda^{2}\right) y\left(y^{2}-1\right)+y \mu_{2} \lambda^{2}+\frac{d}{d r} \log \left(f_{1}\right)-\frac{d}{d r} \log \left(f_{2}\right)
$$

where

$$
f_{2}(y)=1+a_{21} y^{2}+a_{22} y^{4}
$$

with coefficients are given by

$$
\begin{gathered}
a_{21}=2\left(\mu_{1}-\mu_{2}\right) \lambda^{2}-2 \\
a_{22}=1+\lambda^{2}\left(2-\mu_{0}-3 \mu_{1}+6 \mu_{2}\right) / 3+\lambda^{4}\left(-4 \mu_{0}+6 \mu_{1}-2 \mu_{2}-\mu_{0}^{2}-5 \mu_{0} \mu_{2}+3 \mu_{0} \mu_{1}+3 \mu_{1} \mu_{2}\right) / 3
\end{gathered}
$$

For the next member of the superfamily, we show the result of the evaluation of the superpotential, $W_{4}(r)=$ $-\frac{d}{d r} \log \left(\Psi_{0}^{(4)}\right)$ with $\Psi_{0}^{(4)}=a_{3}^{-} a_{2}^{-} a_{1}^{-} \psi_{3}^{(1)}$. It is given by

$$
W_{4}=7 / 2\left(1-\lambda^{2}\right) y\left(y^{2}-1\right)+y \mu_{3} \lambda^{2}+\frac{d}{d r} \log \left(f_{1}\right)+\frac{d}{d r} \log \left(f_{2}\right)-\frac{d}{d r} \log \left(f_{3}\right)
$$

where $f_{1}$ and $f_{2}$ are evaluated in $(29)$ and $(36)$ and $f_{3}$ is set to

$$
f_{3}(y)=1+a_{31} y^{2}+a_{32} y^{4}+a_{33} y^{6}
$$

with the coefficients given by

$$
\begin{aligned}
& a_{31}=3\left(\mu_{2}-\mu_{3}\right) \lambda^{2}+2\left(\mu_{0}-\mu_{1}\right) \lambda^{2}-5 \\
& a_{32}=10+\lambda^{2}\left(6-25 \mu_{0}+15 \mu_{1}-6 \mu_{2}+22 \mu_{3}\right) / 3+ \\
& +\lambda^{4}\left(6 \mu_{0}+2 \mu_{0}^{2}-18 \mu_{1}-6 \mu_{0} \mu_{1}+18 \mu_{2}+13 \mu_{0} \mu_{2}-\right. \\
& \left.-3 \mu_{1} \mu_{2}-6 \mu_{3}-11 \mu_{0} \mu_{3}-3 \mu_{1} \mu_{3}+8 \mu_{2} \mu_{3}\right) / 3 \\
& a_{33}=-10+\lambda^{2}\left(-6+13 \mu_{0}-3 \mu_{1}-12 \mu_{2}-4 \mu_{3}\right)+\lambda^{4}\left(-\frac{148}{15}+\frac{484}{45} \mu_{0}-\frac{206}{45} \mu_{0}^{2}-\frac{84}{5} \mu_{1}+\right. \\
& \left.+\frac{42}{45} \mu_{0} \mu_{1}+2 \mu_{2}+\frac{1}{3} \mu_{0} \mu_{2}-\mu_{1} \mu_{2}-\frac{394}{45} \mu_{3}+\frac{497}{45} \mu_{0} \mu_{3}-\frac{59}{5} \mu_{1} \mu_{3}-\frac{16}{3} \mu_{2} \mu_{3}\right)+ \\
& +\lambda^{6}\left(\frac{8}{3} \mu_{0}+\frac{328}{45} \mu_{0}^{2}-\frac{116}{45} \mu_{0}^{3}-\frac{24}{5} \mu_{1}-\frac{48}{5} \mu_{0} \mu_{1}+\frac{12}{5} \mu_{0}^{2} \mu_{1}+\frac{8}{3} \mu_{2}+\frac{82}{9} \mu_{0} \mu_{2}-\right. \\
& -\frac{14}{9} \mu_{0}^{2} \mu_{2}-6 \mu_{1} \mu_{2}+2 \mu_{0} \mu_{1} \mu_{2}-\frac{8}{15} \mu_{3}-\frac{58}{45} \mu_{0} \mu_{3}+\frac{182}{45} \mu_{0}^{2} \mu_{3}-\frac{2}{5} \mu_{1} \mu_{3}- \\
& \left.-\quad \frac{26}{5} \mu_{0} \mu_{1} \mu_{3}+\frac{8}{9} \mu_{2} \mu_{3}+\frac{44}{9} \mu_{0} \mu_{2} \mu_{3}-4 \mu_{0} \mu_{2} \mu_{3}\right)
\end{aligned}
$$

Casting all the results we have so far for the hierarchy, the following nth-term for the superpotential is induced

$$
W_{n+1}=(n+1 / 2)\left(1-\lambda^{2}\right) y\left(y^{2}-1\right)+y \mu_{n} \lambda^{2}+\frac{d}{d r} \log \left(\frac{\prod_{i=0}^{n} f_{i-1}}{f_{n}}\right) \quad, f_{0}=f_{-1}=1
$$

where $f_{n}(y)$ is a $2 n$-order polynomial of the form

$$
f_{n}(y)=\sum_{i=0}^{n} a_{n i} y^{2 i} \quad, a_{n 0}=1
$$


We stress that since $W_{n+1}$ is a superpotential it checks the Riccati equation,

$$
W_{n+1}^{2}-W_{n+1}^{\prime}=V_{n+1}(r)-\epsilon_{0}^{(n+1)}
$$

where $V_{n+1}(r)$ is the superpartner potential of $V_{n}$ which satisfies

$$
W_{n}^{2}+W_{n}^{\prime}=V_{n+1}(r)-\epsilon_{0}^{n} .
$$

We have therefore a recursive relationship between $W_{n+1}$ and $W_{n}$ given by

$$
W_{n+1}^{2}-W_{n+1}^{\prime}=W_{n}^{2}+W_{n}^{\prime}+\epsilon_{0}^{n}-\epsilon_{0}^{(n+1)}
$$

where $\epsilon_{0}^{n}=-\mu_{n-1}^{2} \lambda^{4}$ and $\epsilon_{0}^{(n+1)}=-\mu_{n}^{2} \lambda^{4}$. After the substitutions we end up with the condition

$$
\begin{aligned}
2 n(1 & \left.-\lambda^{2}\right)^{2} y^{2}\left(y^{2}-1\right)^{2}+\left(y^{2}-1\right) \lambda^{2}\left(\left((2 n-1) \mu_{n-1}-(2 n+1) \mu_{n}\right)\left(1-\left(1-\lambda^{2}\right) y^{2}\right)-2 n\right)+ \\
& +\sum_{i=0}^{n-1} \frac{f_{i-1}^{\prime}}{f_{i-1}}\left(4 \frac{f_{n-1}^{\prime}}{f_{n-1}}-2 \frac{f_{n}^{\prime}}{f_{n}}+2\left(1-\lambda^{2}\right) y\left(y^{2}-1\right)+2 y \lambda^{2}\left(\mu_{n}-\mu_{n-1}\right)\right)+ \\
& +f_{n-1}^{\prime} / f_{n-1}\left(4 n\left(1-\lambda^{2}\right) y\left(y^{2}-1\right)-2 f_{n}^{\prime} / f_{n}+2 y \lambda^{2}\left(\mu_{n}+\mu_{n-1}\right)\right)- \\
& -f_{n}^{\prime} / f_{n}\left(\left(1-\lambda^{2}\right) y\left(y^{2}-1\right)(2 n+1)+2 y \lambda^{2} \mu_{n}\right)+f_{n}^{\prime \prime} / f_{n} \\
& -2 \sum_{i=0}^{n-1} \frac{f_{i-1}^{\prime \prime}}{f_{i-1}}+2 \sum_{i=0}^{n-1}\left(\frac{f_{i-1}^{\prime}}{f_{i-1}}\right)^{2}+\left(2 n\left(1-\lambda^{2}\right)\left(1-3 y^{2}\right)-\lambda^{2}\left(\mu_{n}+\mu_{n-1}\right)\right) d y / d r=0 .
\end{aligned}
$$

where $f^{\prime}=d f / d r$ and $f^{\prime \prime}=d^{2} f / d r^{2}$.

Therefore, $f_{n+1}$ can be determined from the knowledge of $f_{n}$. In this way, the particular cases of $n=1$, $n=2$ and $n=3$ can be checked by inspection and the resulting functions $f_{1}, f_{2}$ and $f_{3}$ perfectly agree with equations $(29),(36)$ and $(38)$ respectively. Notice that the particular case when $\lambda=1$ trivially reduces the $n-$ th term superpotential, equation (39) to $W_{n+1}=y \mu_{n}$, with $\mu_{n}=v-n$, since all the $f$ 's become 1 once all the $a_{i n}$ 's are checked to reduce to zero. The related potentials of the hierarchy are then given by

$$
V_{n+1}(r)=-(v-n)(v-n+1) / \cosh ^{2} r \quad n=0,1, \ldots
$$

This is known as the shape invariant Pöschl-Teller (PT) potential.

\section{Conclusions}

The hierarchy of Hamiltonians is studied for the restricted class of Natanzon potentials, (Ginocchio class), with two parameters and a general form for the superpotential is proposed. The superalgebra drives us to the conclusion that the whole superfamily is a collection of exactly solvable Hamiltonians. The case $\lambda=1$ served as a check of our formulae and was shown to reduce the original potential to the Pöschl-Teller (PT) potential, known to be shape invariant.

As a final remark, the shape invariance concept introduced by Gedenshtein, [12], has motivated several discussions about the exactly solvable potentials. In ref. [8] there is a discussion about this subject which has recently been extended in [14] concerning potentials depending on $n$ parameters . The Natanzon potential is not shape invariant in the usual sense, as most of the exactly solvable potentials are. However, for the restricted class analised here, it was possible to obtain a general form for the superpotential, as shown in the previous section.

The Hulthén potential without the potential barrier term is another example of an exactly solvable potential which is not shape invariant, but for which it is possible to determine a general expression for the superpotential in the hierarchy, [13].

\section{References}

[1] G. A. Natanzon, Theor. Mat. Fiz. 38, 146 (1979).

[2] J. N. Ginocchio, Ann. Phys. 152, 203 (1984).

[3] J. N. Ginocchio, Ann. Phys. 159, 467 (1985).

[4] P. Cordero and S. Salamó, J. Phys A: Math. Gen. 24, 5299 (1991); P. Cordero and S. Salamó, Jafarizadeh; C. Grosche, J. Phys A: Math. Gen. 29, 365 (1996); C. Grosche, J. Phys A: Math. Gen. 29, L183 (1996); S. Codriansky, P. Cordero and Salamó , Nuovo Cimento 112B, 1299 (1997); R. Milson, Int. J. Theor. Phys. 37, 1735 (1998). 
[5] M. A. Jafarizadeh, A. R. Estandyari and H. PanakiTalemi, J. Math. Phys. 41, 675 (2000).

[6] F. Cooper, J. N. Ginocchio and A. Khare, Phys. Rev. D36, 2458 (1987).

[7] G. Levai, D. Baye and J.-M. Sparenberg, J. Phys A: Math. Gen. 30, 8257 (1997).

[8] F. Cooper, A. Khare and U. P. Sukhatme, Phys. Rep. 251, 267 (1995).

[9] C. V. Sukumar, J. Phys. A: Math. Gen. 18, L57 (1985).
[10] C. V. Sukumar, J. Phys. A: Math. Gen. 18, 2917 (1985).

[11] E. Drigo Filho and R. M. Ricotta, Mod. Phys. Lett. A14, 2283 (1989).

[12] L. Gedenshtein, JETP Lett. 38, 356 (1983).

[13] E. Drigo Filho and R. M. Ricotta, Mod. Phys. Lett. A10, 1613 (1995).

[14] J. F. Cariñena and A. Ramos, J. Phys. A: Math. Gen. 33, 3467 (2000). 